\title{
Pregnancy and Peripartum Cardiomyopathy. A Comparative and Prospective Study
}

\author{
Walkiria Samuel Avila, Maria Elisa Carneiro de Carvalho, Cleide K. Tschaen, Eduardo Giusti Rossi, \\ Max Grinberg, Charles Mady, José Antonio Franchini Ramires
}

São Paulo, SP - Brazil

\begin{abstract}
Objective - To assess pregnancy outcome in women with peripartum cardiomyopathy and to compare it with idiopathic cardiomyopathy.

Methods - Twenty-six pregnant women, aged 28.4 26.1 years, with dilated cardiomyopathy were followed. Eighteen patients had peripartum cardiomyopathy [11 with persistent left ventricular systolic dysfunction $(E F=45.2 \pm 2)$ and 7 with recovered ventricular function $(E F=62.3 \pm 3.6)]$. The 8 remaining patients had idiopathic cardiomyopathy $(E F=43.5 \pm 4.1)$. During the prenatal period, limited physical activity and a low-sodium diet were recommended, and hospitalization was recommended when complications occurred.
\end{abstract}

Results - Of the 26 patients, 11 (42.3\%) had a normal delivery; $9(35.5 \%)$ had cardiac complications, $6(22.2 \%)$ had obstetric complications. Two patients (7.7\%) died. Two preterm pregnancies occurred, with 26 health newborns (2 sets of twins). Two miscarriages took place. The cardiac complication rate during pregnancy was lower $(p<0.009)$ in the peripartum cardiomyopathy group without ventricular dysfunction and greater $(p=0.01)$ in the idiopathic group when compared with the peripartum group with ventricular dysfunction. Changes in left ventricular ejection fraction were not observed $(p<0.05)$ in the postpartum period, when compared with that during pregnancy in the 3 groups.

Conclusion - Pregnancy in patients with dilated cardiomyopathy is associated with maternal morbidity. Left ventricular function is a prognostic factor and must be the most parameter when counseling patients with peripartum cardiomyopathy about a new pregnancy.

Keywords: peripartum cardiomyopathy, pregnancy, maternal complication, fetal complication

Instituto do Coração do Hospital das Clínicas - FMUSP

Mailing address: Walkiria Samuel Avila - InCor - Av. Dr. Enéas C. Aguiar, 44 05403-000 - São Paulo, SP - Brazil - E-mail: walkiria@incor.usp.br
Peripartum cardiomyopathy is a rare disease of unknown cause that affects women of reproductive age. Its incidence is related to the peripartum period. Hypotheses of its cause are focused on the physiologic relationship between pregnancy and the postpartum period and infective genetic disorders and hormonal and metabolic changes ${ }^{1-3}$.

The criteria for diagnosis of peripartum cardiomyopathy include heart failure in the last month of pregnancy or within the first 5 postpartum months, in the absence of a determinable cause of cardiac failure or the absence of demonstrable preexisting heart disease ${ }^{4}$, and systolic dysfunction confirmed by a lower ejection fraction (EF) or the left ventricular fractional shortening, or both of these, shown by echocardiographic measurement ${ }^{5}$.

Studies ${ }^{6-8}$ about the natural cause of peripartum cardiomyopathy estimate that more than half of these patients experience a regression in ventricular dysfunction, while about $25 \%$ evolve to death within 3 months due to heart failure, arrhythmias, or thromboembolism and the remaining patients develop dilated cardiomyopathy.

No consensus, therefore, exists regarding recommendations for future pregnancies in women who have peripartum cardiomyopathy. The persistence of ventricular dysfunction is associated with a high risk of complications and maternal death. On the other hand, the recovery of ventricular function does not assure a good prognosis of the next pregnancy, in addition to the hypotheses about the recurrence of the disease, decreased $\mathrm{EF}$, and heart failure in the peripartum period ${ }^{9,10}$.

We undertook a study to evaluate the clinical and obstetric evolvement of pregnancy in women with a previous diagnosis of peripartum cardiomyopathy, to evaluate the factors associated with its prognosis, and to compare it with the evolvement of patients with idiopathic dilated cardiomyopathy.

\section{Methods}

From 1990 to 1998,26 pregnant women were followed. All had dilated cardiomyopathy discovered during routine 
evaluation at the Instituto do Coração (InCor) (Heart Institute). Eighteen presented with peripartum cardiomyopathy, and 8 presented with idiopathic dilated cardiomyopathy. Of the 18 patients with peripartum cardiomyopathy, 11 had persistent left ventricular systolic dysfunction $(\mathrm{EF}=$ $45.2 \pm 2 \%$ ), whereas 7 patients recovered ventricular function $(\mathrm{EF}=62.3 \pm 3.6 \%)$, prior to pregnancy in the study. Mean EF in the idiopathic dilated cardiomyopathy group was $43.5 \pm 4.1 \%$. Diagnosis of cardiomyopathy and classification in peripartum cardiomyopathy and idiopathic dilated cardiomyopathy followed the criteria adopted by the World Health Organization Committee, defined in 1980 and modified in $1996^{11}$. We performed either clinical, echocardiographic, or hystologic examinations, or all of these.

The mean age of patients with peripartum cardiomyopathy and ventricular dysfunction was $26 \pm 7$ years. Of those patients without ventricular dysfunction, it was $26 \pm 6,1$. The mean age for those patients with idiopathic dilated cardiomyopathy was $29.5 \pm 6.2$ (tab. I). All patients were in functional class I/II (NYHA) at the beginning of pregnancy, and all had a history of heart failure and decreased EF on echocardiography, except for 2 patients, 1 with peripartum cardiomyopathy and the other with idiopathic dilated cardiomyopathy.

Twenty patients had electrocardiographic alterations (right branch conduction disturbance, supraventricular and ventricular extrasystoles, left ventricular hypertrophy) that were equally distributed between the groups with ventricular dysfunction (tab. I).

In patients with peripartum cardiomyopathy, the elapsed time between the onset of the disease and the studied pregnancy was, on average, 52.6 months for those patients that recovered ventricular function and 55.8 months for the remaining patients.

Prenatal care included periodic appointments with a cardiologist and an obstetrician, a control ECG, and echocardiograms during pregnancy and within 3 months after the delivery. We recommended that patients refrain from physical exercise and begin a diet low in sodium after the second trimester of pregnancy, and we also recommended hospitalization when cardiac and obstetric complications occurred. Medications previously prescribed, such as digitalis, diuretics, and antiarrhythmic drugs, were continued, except for angiotensin-converting enzyme inhibitors and anticoagulants, respectively, replaced by hydralazine and heparin, according to the Cardiopathy and Pregnancy Consensus of the Brazilian Society of Cardiology ${ }^{12}$.

Eight patients became pregnant while taking captopril, which was suspended at the first routine prenatal evaluation, which occurred in the majority of case during the first trimester of pregnancy. Four patients continued taking amiodarone during pregnancy, which was considered necessary for the control of arrhythmias.

An analysis of the occurrence of clinical events (heart failure, thromboembolism, cardiac arrhythmia, and death), and of ECG, and EF changes before and after parturition was performed by calculating minimum, maximum, and median values, and standard deviation, for quantitative variables. Tables of absolute and relative frequencies were used for the qualitative variables. The comparative analysis was performed on 3 subgroups: peripartum cardiomyopathy with ventricular dysfunction, peripartum cardiomyopathy without ventricular dysfunction, and idiopathic dilated cardiomyopathy. Pearson $\mathrm{c}_{2}$ tests were used to analyze functional class, electrocardiographic alterations, use of medication and maternal-fetal complications. Fisher's exact test and the Student $t$ test were used for age, ejection fraction, and fetal and maternal death variables. A probability value lower than 0.05 was considered significant.

\section{Results}

Clinical and obstetrical evolvement is shown in table II. Of the 26 patients, 11 (42.3\%) had a normal delivery; 9 (35.5\%) had cardiac complications, and 6 (22.2\%) had obstetric complications. Of the cardiac complications, 8 resulted from heart failure, 2 from sustained ventricular tachycardia, and 1 from thromboembolism. Two maternal deaths occurred: one due to a retained abortion, infection, and heart failure in a patient with idiopathic dilated cardiomyopathy and another because of heart failure in a patient with peripartum cardiomyopathy with ventricular dysfunction. Obstetric complications were 2 miscarriages, 1 case of gestational diabetes, and 3 cases of urinary infection. The 26 healthy newborns included twins, without malformations related to maternal cardiomyopathy or to the type of therapeutic regimen adopted.

During pregnancy, 7 patients did not take cardiovascular drugs, whereas 21 patients needed medication, such

\begin{tabular}{|c|c|c|c|c|c|c|c|c|c|c|c|c|}
\hline \multirow[b]{2}{*}{ Etiology } & \multirow{2}{*}{$\begin{array}{c}\text { Age } \\
\text { (years) }\end{array}$} & \multirow[b]{2}{*}{$\Delta \mathrm{T}$} & \multirow[b]{2}{*}{$\mathrm{EF}(\%)$} & \multicolumn{3}{|c|}{ Parity } & \multicolumn{4}{|c|}{ FC (NYHA) } & \multicolumn{2}{|c|}{ ECG Alteration } \\
\hline & & & & 1 & 2 & $>3$ & I & II & III & IV & Yes & No \\
\hline $\begin{array}{l}\mathrm{CMP}(11) \\
\mathrm{EF}<55 \%\end{array}$ & $26 \pm 7$ & 55.8 & $45.2 \pm 2$ & 0 & 4 & 7 & 1 & 9 & 1 & 0 & 9 & 2 \\
\hline $\begin{array}{l}\mathrm{PCM}(7) \\
\mathrm{EF}>55 \%\end{array}$ & $26 \pm 6.1$ & 52.6 & $62.3 \pm 3.6$ & 0 & 4 & 2 & 2 & 5 & 0 & 0 & 2 & 5 \\
\hline IDC (8) & $29.5 \pm 6.2$ & 36.6 & $43.5 \pm 4.1$ & 3 & 0 & 5 & 1 & 6 & 1 & 0 & 4 & 4 \\
\hline
\end{tabular}




\begin{tabular}{|c|c|c|c|c|c|c|}
\hline & Age & Etiology & $\mathrm{EF}$ & Maternal complication & NB Weight & EF PP \\
\hline 1 & 28 & IDC & 29 & Retained abortion/death & & 24 \\
\hline 2 & 26 & $\mathrm{PC}$ & 58 & Diabetes & 3090 & 54 \\
\hline 3 & 31 & IDC & 32 & CHF & 3380 & 32 \\
\hline 4 & 35 & PNL & 75 & Syncope/arrhythmia & 3580 & 67 \\
\hline 5 & 16 & PCVD & 43 & & 3870 & 45 \\
\hline 6 & 36 & PCVD & 41 & & 3150 & 75 \\
\hline 7 & 26 & PCVD & 51 & & 2220 & \\
\hline 8 & 25 & PCVD & 41 & & 3060 & 31 \\
\hline 9 & 26 & $\mathrm{PC}$ & 69 & & 3520 & \\
\hline 10 & 19 & IDC & 54 & IGT & $2900 / 2910$ & 55 \\
\hline 11 & 38 & IDC & 33 & Abortion & & 34 \\
\hline 12 & 24 & $\mathrm{PC}$ & 59 & & 3680 & \\
\hline 13 & 23 & PCVD & 48 & IGT & 2860 & 41 \\
\hline 14 & 27 & PCVD & 44 & IGT/death/CHF & 3260 & 56 \\
\hline 15 & 23 & $\mathrm{PC}$ & 56 & & 3110 & \\
\hline 16 & 28 & IDC & 35 & $\mathrm{CHF}$ & 2150 & 46 \\
\hline 17 & 15 & $\mathrm{PC}$ & 60 & & 3920 & \\
\hline 18 & 30 & PCVD & 50 & $\mathrm{CHF}$ & 2950 & \\
\hline 19 & 29 & $\mathrm{PC}$ & 59 & & 3520 & \\
\hline 20 & 41 & PCVD & 44 & & 3149 & \\
\hline 21 & 31 & IDC & 57 & $\mathrm{CHF}$ & 3140 & 66 \\
\hline 22 & 23 & PCVD & 53 & & 2230 & 73 \\
\hline 23 & 23 & PCVD & 38 & $\mathrm{CHF}$ & 3500 & \\
\hline 24 & 36 & IDC & 50 & $\mathrm{CHF}$ & 3140 & 40 \\
\hline 25 & 38 & IDC & 33 & $\mathrm{CHF}$ & 2950 & 47 \\
\hline 26 & 33 & PCVD & 44 & $\mathrm{CHF} / \mathrm{PT}$ & $2300 / 2500$ & 44 \\
\hline
\end{tabular}

as digitalis, hydralazine, heparin or amiodarone, or both, for prevention or treatment of complications.

A comparative analysis between the 3 groups, regarding age, parity, and functional class in the beginning of pregnancy were not different $(p<0.05)$. Of the cases of peripartum cardiomyopathy with ventricular dysfunction and idiopathic dilated cardiomyopathy, no differences existed regarding age $(\mathrm{p}=0.06)$, functional class at the beginning of pregnancy $(\mathrm{p}=0.37)$, electrocardiogram alterations $(\mathrm{p}=0.38)$, $\mathrm{EF}(\mathrm{p}=0.06)$, use of captopril $(\mathrm{p}=1.0)$, use of amiodarone $(\mathrm{p}=0.59)$, and fetal death $(\mathrm{p}=0.59)$ (tab. II).

A cardiac complication rate during gestation was lower $(p<0.009)$ in the peripartum cardiomyopathy without ventricular dysfunction subgroup and higher in the idiopathic dilated cardiomyopathy subgroup, when compared with that in the peripartum cardiomyopathy with ventricular dysfunction subgroup $(p=0.01)$. A comparative analysis of 17 records within the first 3 months after delivery showed that no significant changes occurred between the electrocardiogram $(p>0.05)$ and $\mathrm{EF}(\mathrm{p}>0.05)$ after delivery when compared with that in the pregnancy records in the 3 groups (tab. III).

\section{Discussion}

Diversity in the clinical features of peripartum cardiomyopathy causes difficulties in making recommendations about future pregnancies, when the factors of its prognosis are still not clearly established.
This prospective study examined the evolvement of pregnancy in patients who had experienced peripartum cardiomyopathy in a previous pregnancy, with or without recovery of ventricular function, and compared this with the evolvement of patients with idiopathic dilated cardiomyopathy, observing similarities in age, functional capacity, and electrocardiographic and $\mathrm{EF}$ alterations in the echocardiogram.

Results showed that clinical evolvement of pregnancy in patients with peripartum cardiomyopathy who recover ventricular function was favorable, and patients did not ex-

\begin{tabular}{|c|c|c|c|c|}
\hline \multirow[b]{2}{*}{ Groups } & \multicolumn{2}{|c|}{ Peripartum cardiomyopathy } & \multirow{2}{*}{$\begin{array}{c}\text { Idiopathic } \\
\text { Cardiomyopathy }\end{array}$} & \multirow[t]{2}{*}{$\mathrm{p}$ value } \\
\hline & $\begin{array}{c}\text { With } \\
\text { dysfunction }\end{array}$ & $\begin{array}{c}\text { Without } \\
\text { dysfunction }\end{array}$ & & \\
\hline Age (years) & $26 \pm 7$ & $26 \pm 6.1$ & $29.5 \pm 6$ & NS \\
\hline $\begin{array}{l}\text { EF before } \\
\text { pregnancy (\%) }\end{array}$ & $45.2 \pm 2 *$ & $62.3 \pm 3.6$ & $43.5 \pm 4.1^{*}$ & NS* \\
\hline $\begin{array}{l}\text { Cardiac } \\
\text { complication }\end{array}$ & $3(27.3 \%)^{* *}$ & $1(14.2 \%)$ & $5(62.5 \%)^{* *}$ & $\mathrm{p}=0.01 * *$ \\
\hline Maternal death & $1(9.1 \%)$ & - & $1(14.2 \%)$ & NS \\
\hline \multicolumn{5}{|c|}{ postpregnancy $(\%)$} \\
\hline \multicolumn{5}{|c|}{$\begin{array}{l}\text { EF- left ventricle ejection fraction; } * \mathrm{p}>0.05 \text { - comparative analysis between } \\
\mathrm{EF} \text { of peripartum cardiomyopathy with dysfunction and idiopathic dilated } \\
\text { cardiomyopathy; } * * \mathrm{p}<0.05 \text { - comparative analysis regarding the incidence } \\
\text { of maternal cardiac complication of peripartum cardiomyopathy with dys- } \\
\text { function and idiopathic dilated cardiomyopathy; } * * * \mathrm{p}>0.05 \text { - comparative } \\
\text { analysis between EF before and after pregnancy in the } 3 \text { groups. }\end{array}$} \\
\hline
\end{tabular}


perience complications regarding cardiopathy. Additionally, EF was maintained at the same level as that observed within the first 3 months after delivery.

These data are in accordance with those from the studies performed by Albanesi Filho et al ${ }^{13}$ and Sutton et al ${ }^{14}$ who showed in a prospective analyses good evolvement, absence of mortality, or recurrence of peripartum cardiomyopathy in 11 pregnancies of women who had recovered ventricular function after a diagnosis of peripartum cardiomyopathy.

On the other hand, our results are not in accordance with those of Elkayam et $\mathrm{al}^{9}$ who found a reduction of $8 \%$ in EF in the postpartum period in women with peripartum cardiomyopathy, considered as having recovered from ventricular dysfunction, and who began pregnancy with mean EF of $56 \pm 7 \%$. In addition, we cannot agree with the authors' assumption that the recurrence of peripartum cardiomyopathy following pregnancy was based on a reduction in EF after delivery. It is possible that bias in the selection of the retrospective cohort, in the criteria of the diagnosis of the disease, and in ventricular dysfunction are reasons why the results of the studies do not correlate.

Lampert et al ${ }^{15}$ demonstrated that 7 patients with peripartum cardiomyopathy, who regained normal resting left ventricular size and performance have decreased contractile reserve revealed by the use of dobutamine. These data and controversies reported in the literature emphasize the need to revise current criteria of ventricular function recovery in peripartum cardiomyopathy. Perhaps current parameters based on conventional echocardiographic analysis are not enough to ensure a diagnosis of normal ventricular function in women with a previous history of peripartum cardiomyopathy.

On the other hand, a mean clinical follow-up of 8.6 years of 42 women with peripartum cardiomyopathy showed that the $75 \%$ of patients that recovered ventricular function did not have any kind of limitation in functional capacity and had a good quality of life ${ }^{16}$.

Another important aspect of the natural history of peripartum cardiomyopathy is the expectation of a better prognosis because of advances in the therapeutics of heart failure in recent decades. Indeed, the $50 \%$ rate of ventricular function recovery reported in the $1970 \mathrm{~s}^{3}$ is below the $75 \%$ reported in recent publications ${ }^{16}$. Additionally, mortality rates among patients that remained with dilated cardiomyopathy also decreased from $85 \%$ to $30 \%$ because of the benefits of cardiac transplantation ${ }^{17}$ and to the strategies of drug therapy for this group of patients ${ }^{18}$.

Thus, greater life expectancy in short-term and longterm follow-up and the good evolvement of a subsequent pregnancy ${ }^{13-15}$ do not justify ruling out pregnancy in women who experienced peripartum cardiomyopathy in a previous pregnancy, but who recovered left ventricular function. Prospective studies, with a greater cohort and assessment of ventricular function are necessary to define the correct counseling for these women.
On the other hand, our study showed that the cardiac complication rate in women with peripartum cardiomyopathy with persistent ventricular dysfunction, including 1 maternal death, highlight the high risk in this group of patients. However, no signs of worsening of ventricular dysfunction were estimated through postpartum EF or signs of recurrence of the disease even in the 2 sets of twins, a recognized factor of predisposition to peripartum cardiomyopathy ${ }^{3}$.

Hemodynamic overload after the first trimester of pregnancy, demonstrated by a $10 \%$ increase in final left ventricular diastolic volume and a $45 \%$ increase in cardiac output ${ }^{19}$ worsens left ventricular systolic function, which usually reverses after delivery.

However, we believe that this circulatory response is ventricular dysfunction-dependent and not cause-dependent. Therefore, the remaining question is whether or not peripartum cardiomyopathy with ventricular dysfunction persistence has a natural history similar to that of other causes of dilated cardiomyopathy, including pregnancy ${ }^{20}$.

In this regard, our results corroborate those of previous studies that had similar patterns regarding obstetric and cardiologic evolvement between peripartum cardiomyopathy and idiopathic dilated cardiomyopathy. It is worth mentioning that among clinical, echocardiographic, and hemodynamic variables previously shown ${ }^{20,21}$, hystologic findings of myocarditis in endomyocardial biopsies was controversial ${ }^{22,23}$, and in some series it was significantly higher in peripartum cardiomyopathy ${ }^{24}$.

An additional factor of maternal fetal morbidity is the suitability of therapeutics during pregnancy in patients with dilated cardiomyopathy. Sodium and physical activity restrictions in association with drugs like digoxin and furosemide, which are not contraindicated in this period, help control heart failure during pregnancy. However, use of angiotensin-converting enzyme inhibitors is associated with side effects, such as oligohydramnios, delay in intrauterine development, prematurity, fetal renal failure, bone malformation, and neonatal death ${ }^{25}$. In our study, these effects were not identified in 8 patients who became pregnant while taking captopril; however, this medication was replaced in the first trimester, as soon as pregnancy was confirmed.

Hydralazine, with or without nitrates, as an alternative to angiotensin- converting enzyme inhibitors was based on the results of the V-HeFT-II study ${ }^{26}$ that demonstrated enhancement in functional capacity of oxygen consumption, and of EF in a 12-month period-equivalent to the peripartum period. The experience with hydralazine in the treatment of hypertensive disease due to pregnancy shows that no obstetric and fetal contraindication exists regarding its use, during any phase of pregnancy ${ }^{27}$.

Ventricular arrhythmias are usually complex and are related to death in patients with dilated cardiomyopathy, therefore, requiring effective control with antiarrhythmic drugs like amiodarone. However, this medication may be toxic for mother and baby, leading to hypothyroidism and retarded growth, with the risk of perinatal death ${ }^{28}$. In our study, we continued to prescribe amiodarone at reduced dosages (up 
to $200 \mathrm{mg}$ /day) in 4 patients that already used it, because we considered it essential for controlling complex ventricular arrhythmias, and we did not observe side effects in the baby.

Systemic or pulmonary thromboembolism is another frequent complication, described in more than half of the cases of peripartum cardiomyopathy with ventricular dysfunction. During pregnancy and the postpartum period, hypercoagulability, including activation of coagulation factors, increase in plasma fibrinogen, and platelet adhesion, increases the risk of thrombosis, which is aggravated by the need for prolonged rest due to congestive heart failure. Because of this, patients with dilated cardiomyopathy with ventricular dysfunction must be anticoagulated with heparin at prophylactic doses, because this medication does not cross the placenta.

In summary, peripartum cardiomyopathy has been referred to as a distinct entity from other dilated cardiomyopathies because of its relation to the peripartum period and the peculiarities of its natural history. Evolvement of pregnancy in patients with peripartum cardiomyopathy with ven- tricular dysfunction was not different when compared with that of the idiopathic dilated cardiomyopathy group. However, patients who recovered ventricular function did not experience complications, which is similar to the experience of women considered to be healthy. The present study reveals the limitations inherent in a small cohort and the conventional methods of ventricular function assessments. However, because it is a rare disease, this prospective analysis may add to the data about a very controversial subject.

Our results enabled us to conclude that left ventricular function is the determinant factor in pregnancy following the diagnosis of peripartum cardiomyopathy. Based on our understanding, discouragement of a new pregnancy must be reserved for patients with peripartum cardiomyopathy who have ventricular dysfunction.

\section{Acknowledgments}

To Dr. Maeve de Barros Correia for the valuable suggestions given in the review of the text.

\section{References}

1. Pearson GD, Veille JC, Rahimtoola S, et al. Peripartum cardiomyopathy. JAMABrazil 2000; 283: 1183-8.

2. Veille J,Zaccaro D. Peripartum cardiomyopathy: summary of international survey on peripartum cardiomyopathy. Am J Obstet Gynecol 1999; 181: 315-9.

3. Homans DC. Peripartum cardiomyopathy. N Eng J Med 1985; 312: 1432-6.

4. Demakis JG, Rahimtoola SH. Peripartum cardiomyopathy. Circulation 1971; 44: 964-8.

5. LampertMB,Lange RM. Peripartum cardiomyopathy. AmHeart J 1995; 130:860-70.

6. Demakis JG, Rahimtoola SH, Sutton GC, et al. Natural course of peripartum cardiomyopathy. Circulation 1971; 44: 1053-61.

7. Veille J. Peripartum cardiomyopathies: a review. Am J Obstet Gynecol 1984; 148: 805-17.

8. Souza JL, Carvalho FC, Nastari L, Mady CC. Left ventricular function after new pregnancy in patients with peripartum cardiomyopathy. J Card Fail 2001; 7: 30-5.

9. Elkayam U, Tummala PP, Rao K, et al. Maternal and fetal outcomes of subsequent pregnancies in women with peripartum cardiomyopathy. N Engl J Med 2001; 344: 1567-71.

10. Ceci O, Berardesca C, Caradonna F, et al. Recurrent peripartum cardiomyopathy. Eur J Obstet Gynecol and Reprod Biol 1998; 76: 29-30.

11. Richardson P, McKenna W, Bristow MR, et al. Report of the 1995 World Health Organization/International Society an Federation of Cardiology Task Force on the definition and classification of cardiomyopathies. Circulation 1996; 93: 841-2.

12. Consenso do Departamento de Cardiopatia e Gravidez da Sociedade Brasileira de Cardiologia. Arq Bras Cardiol, 1999; 72(supl III): 8.

13. Albanesi $\mathrm{F}^{\circ} \mathrm{FM}$, Silva TT. O comportamento das gestações subseqüentes na cardiomiopatia periparto. Arq Bras Cardiol 1999; 73: 47-52.

14. Sutton MSJ, Cole P, Plappert M, et al. Effects of subsequent pregnancy on left ventricular function in peripartum cardiomyopathy. Am Heart J 1991; 121; 1776-8.

15. Lampert MB, Weinert L, Hisbbard J, et al. Contractile reserve in patients with peripartum cardiomyopathy and recovered left ventricular function. Am J Obstet Gynecol 1997; 176: 189-95.
16. Felker GM, Jaeger CJ, Klodas E, et al. Myocarditis and long-term survival in peripartum cardiomyopathy. Am Heart J 2000; 140: 785-91.

17. Carvalho AG, Almeida D, Cohen M, et al. Successful pregnancy, delivery and puerperium in a heart transplant patient with previous peripartum cardiomyopathy. Eur Heart J 1992; 13: 1589-91.

18. The SOLVD Investigators. SOLVD treatment study. Am JCardiol 1990; 66:315-22.

19. Robson SC, Hunter S, Boys RJ, Dunlop W. Serial study of factors influencing changes in cardiac output during human pregnancy. Am J Physiol 1989; 226: H1060-5.

20. Felker GM, Hu W, Hare JM, et al. The spectrum of dilated cardiomyopathy: the Johns Hopkins Experience with 1,278 patients. Medicine 1999; 78: 270-83.

21. Chan L, Hill D. Echocardiography for peripartum cardiomyopathy. Am JEm Med 1999; 17: 578-80

22. Rizeq MN, Rickenbacher PR, Fowler MB, et al. Incidence of myocarditis in peripartum cardiomyopathy. Am J Cardiol 1994; 74: 474-7.

23. Mady C, Barretto ACP, Belotti G, et al. Biópsia endomiocárdica em pacientes portadoras de miocardiopatia periparto. Arq Bras Cardiol 1986; 47: 403-5.

24. O'Connell JB, Constanzo O, Nordin MR, et al. Peripartum cardiomyopathy: Clinical, hemodynamic, histologic and prognostic characteristics. J Am Coll Cardiol 1986; 8: 52-6

25. Pryde PG, Thorpe SS, Lamont CA. Angiotensin-converting enzyme inhibitor fetopathy. J Am Soc Nephrol 1993; 3: 1575-82.

26. Chon JN, Johnson G, Zeische S, et al. A comparison of enalapril with hydralazine-isosorbide dinitrate in the treatment of chronic congestive heart failure. $\mathrm{N}$ Engl J Med 1991; 325: 303-10.

27. Paterson-Brown S, Robson SC, Redfern N, Walkinshaw SA, Swiet M. Hydralazine boluses for the treatment of severe hypertension in preeclampsia. Br J Obstet Gynaecol 1994; 101: 409-15.

28. De WolfD, De Schepper J, Verhaaren H, et al. Congenital hypothyroid goiter and amiodarone. Acta Paediatr Scand 1988; 77: 616-8. 